\title{
¿Que Vuelva la Política al Análisis Comunicológico! (consecuencias operativas de la Epistemología aplicada)
}

Let the Policy Return to Communicological Analysis! (Operational consequences of applied Epistemology)

Deixe a política voltar à análise comunicológica (consequências operacionais da epistemologia aplicada)

Roberto Agustín FOLLARI, Argentina

Universidad Nacional de Cuyo / rfollari@gmail.com

Chasqui. Revista Latinoamericana de Comunicación

N. ${ }^{\circ} 141$, agosto-noviembre 2019 (Sección Tribuna, pp. 17-30)

ISSN 1390-1079 / e-ISSN 1390-924X

Ecuador: CIESPAL

Recibido: 25-02-2019/Aprobado: 10-07-2019 


\title{
Resumen
}

Las ciencias de la Comunicación -como todas las ciencias sociales- carecen en paradigmas, en el estricto sentido de Kuhn. A esta falta de acuerdo conceptual se suma la carencia de un objeto teórico propio, siendo un espacio transitado por el aporte de varias y diversas disciplinas. Ello debilita la identidad disciplinar, la cual en A.Latina se ha constituido en gran parte por vía de los estudios culturales. Por esto se ha enfatizado lo cultural por sobre lo político en los estudios del área, lo cual es altamente problemático cuando las fakenews se han desplegado ampliamente, y cuando una política de limitación al ejercicio arbitrario de los medios hegemónicos se hace cada vez más necesaria.

Palabras clave: epistemología de la comunicación, paradigma, ciencias sociales, política

\begin{abstract}
Communication sciences -as every social sciences- have no paradigms, in Kuhn's sense. This impossibility for conceptual according adds to lack about having one own theoretical object, because of that it's a space where diverse disciplines transit. This weakens disciplinar identity, which has been constitued mainly by cultural studies in Latin America. The result is that Communication sciencies have emphasized cultural problematic over political one, and that is a heavy problem when fakenews have strongly appeared, and when a politics for controlling hegemonic media is more and more neccesary.
\end{abstract}

Keywords: epistemology of communication, paradigm, social sciences, politics

\section{Resumo}

As ciências da comunicação - como todas as ciências sociais - carecem de paradigmas, no sentido estrito de Kuhn. A essa falta de acordo conceitual se soma a falta de um objeto teórico próprio, sendo um espaço percorrido pela contribuição de diversas e diversas disciplinas. Isso enfraquece a identidade disciplinar, que em A. Latina foi constituída em grande parte por meio de estudos culturais. Por esse motivo, o cultural tem sido enfatizado sobre o político nos estudos da área, o que é altamente problemático quando os fakenews são amplamente utilizados e quando uma política de limitação ao exercício arbitrário de meios hegemônicos se torna cada vez mais necessária.

Palavras-chave: epistemologia da comunicação, paradigma, ciências sociais, política 


\section{Introducción}

No hay paradigmas en las ciencias sociales. Es una afirmación nada aventurada, pues ya la sostuvo Kuhn (1980) desde su obra fundamental; pero todavía aparece como una novedad, frente al uso indiscriminado y libérrimo de la noción de "paradigma", abusivamente presente en muchísimos desarrollos de las ciencias sociales. Lo hemos planteado hace ya muchos años (Follari, 2000), pero ciertamente de poco sirven ciertas precisiones conceptuales frente a la avalancha impuesta mayoritariamente, que no hace caso de lo epistemológico para sostenerse.

Hacer esta afirmación sobre la inexistencia de paradigmas en estudios sobre lo social, no implica una especie de "preciosismo teoricista": tal delimitación sirve para establecer las diferencias con las ciencias físico-naturales, y por vía de ello, asentar con mayor claridad las características epistémicas de nuestras propias producciones.

De tal modo, podemos asumir que el acuerdo entre teorías -ya que no paradigmas- es imposible en nuestras ciencias, como también lo es, el que alguien pudiera imaginar entre diferentes paradigmas (en ese caso sucesivos) dentro de las ciencias físico-naturales. La relación entre esas diferentes teorías (al interior de una misma y sola disciplina) es una relación de mutua inconmensurabilidad, en los términos disímiles en que el mismo Kuhn, (1980) fijó en dos momentos: al comienzo, como imposibilidad de mutua comunicación y entendimiento y después -ya cerca de su muerte- como relación hermenéutica de no/continuidad entre diferentes horizontes de comprensión (Kuhn, 1989). La segunda noción es la que se implica una posibilidad parcial de entendimiento y comunicación, si bien siempre irreductible al acuerdo sobre esos parámetros desde los que se organiza tanto la explicación conceptual, como la mirada misma sobre el material empírico y los procedimientos metodológicos que la posibilitan.

Lo dicho implica una condición de fuerte desventaja frente a las ciencias físico-naturales: estas acuerdan -cada una dentro de su propia "comunidad científica"- acerca de teoría, modos de la investigación y hasta procesos de formación de los nuevos miembros de esa "comunidad" 1 . Y por tanto, la condición teórica y metodológica de esa disciplina puede "naturalizarse". Eso es, precisamente, el paradigma: una modalidad determinada de comprender los fenómenos que se impone hasta aparecer como "la" mirada, como la única posible, casi como lo que lo real mismo dicta y establece.

Tal "efecto de verdad naturalizada" es lo que no puede lograrse en ciencias sociales. El desacuerdo es constitutivo entre nuestras teorías. Y ello, no porque

1 Ciertamente que la noción de "comunidad científica" plantea varios problemas (sin que ello impida advertir su utilidad), tal el implícito de lo común/itario, un tanto idealista -si bien Kuhn siempre planteó el conflicto dentro de tales "comunidades"-; y también los límites, ¿quiénes están habilitados para ser miembros de la comunidad? ¿cuál es el umbral para acceder a ella? (Kuhn, T. 1989). 
algunas de nuestras concepciones sean imposibles de someterse al célebre "experimento crucial" que imponía el empirismo, desde los neopositivistas a Popper. Es por una razón más sutil: si bien podemos entender que exista algún nivel de presencia, de ideología en las ciencias físico-naturales (por ej. en lo que imponen los procesos de financiamiento, en la elección temática, en los usos de lo investigado), esto es más flagrante en las ciencias sociales. Postulamos (y no podemos desarrollarlo aquí) que la imposibilidad de acuerdo ideológico -una homogeneización en ese plano es imposible- conlleva la imposibilidad de acuerdo teórico, en tanto las ideologías son constitutivas de las tomas de partido teóricas en ciencias sociales. Dicho de otro modo, si bien no creemos que a cada ideología corresponda una y sólo una teoría -no hay tal "correspondencia dura" entre teorías e ideologías-, sí estamos convencidos de que cada ideología establece límites en cuanto a las teorías que le son compatibles. De tal modo, el espacio de inteligibilidad que sobre lo social establece cada una de las ideologías (en este caso destacamos las "ideologías políticas"), opera como suelo silencioso pero necesario de las producciones teóricas, así como de las "elecciones de teoría" que hace cada uno de los investigadores sociales -elecciones inevitables, dado que al no existir paradigma, la teoría no está ya supuesta y naturalizada, como ocurre en ciencias físico-naturales-.

Bien, entonces: nuestras ciencias, las sociales, son -hasta algún punto- una inevitable serie de torres de Babel, donde co-existen (en todos los casos, y para cada una de las ciencias diferencialmente) teorías con lenguajes diferentes, con sus disímiles protocolos de explicación, de metodología para la investigación, y de categorías para la visualización e interpretación de lo empírico.

Esto es, ciertamente, lo que motiva algún desprecio que desde las ciencias físico-naturales se suele tener hacia las nuestras. Es que, a nivel de la descripción, pareciera que estuviéramos comparando disciplinas que no usan teoría y van directamente a (eso imposible que es) "lo real mismo", con nuestras ciencias, que son una multiplicidad de puntos de vista diferenciados e irreconciliables, en tanto irreductibles a la unicidad de criterios.

Por cierto que la filosofía kuhniana muestra claramente cómo las ciencias físico-naturales, al naturalizar su mirada dentro del paradigma, no advierten que sí usan teoría, y que su punto de vista es momentáneo, contingente y modificable (Follari, 200o). Pero no es eso lo que aquí nos interesa, sino advertir cómo, en ciencias de la comunicación (o "ciencia" de la comunicación en singular, si alguien así lo prefiere) esta condición desventajosa de las ciencias sociales está multiplicada; es decir, está aún más comprometida en la pluralidad in-conclusiva de puntos de vista múltiples e inconmensurables.

Es que, a diferencia de la sociología -que produjo desde lo académico una ciencia consolidada que luego dio lugar a una profesión, esta última no muy bien delineada-, en comunicación el camino fue al revés: algunas profesiones 
previamente existentes (especialmente la de periodista/s) conllevaron la necesidad de legitimarse y conceptualizarse a nivel científico (Follari, 2003)2.

Ello lleva a que en Comunicación partamos desde ciertos quehaceres de los cuales hay que dar razón: se diría que nos encontramos ante un objeto real, asumiendo el ya multi-aceptado repertorio conceptual de P.Bourdieu (1975). No, prioritariamente, ante un "objeto teórico", que hubiera sido delimitado a partir de la construcción de un punto de vista desde lo académico/teórico. El resultado de ello es que, si atendemos a que cualquier real puede ser leído desde muchas miradas, lo comunicológico requiere de múltiples aproximaciones que bien conocemos: Sociología, Economía, Antropología, Teoría Política, Lingüística, Semiótica...

Es decir, lo original en comunicación no es producir teorías desde cero, sino combinar teorías previas y hacerlas producir acercamientos y síntesis nuevos, respecto de un objeto real propio que, no cabe dudas, más de una vez ha resultado imposible circunscribir, en tanto la multiplicidad de acercamientos dispersa inevitablemente los puntos de mirada.

Tenemos, entonces, un número mayor de teorías que se ponen en curso que en muchas de las demás ciencias sociales (Martino, 2007); y menos definición específica del objeto de análisis. Ello no es una desgracia metafísica que se hubiera precipitado sobre comunicación, dice, simplemente, de su singularidad epistémica, que -por cierto- es análoga a la de otras disciplinas sociales, entre ellas Ciencias de la Educación y Trabajo Social.

\section{De la dificultad epistemológica a la vacilación conceptual}

Dentro de estas ciudadanías comunicativas está la que me interesa aquí: las ciudadanías celebrities. Esas que desarrollan el querer estar en las pantallas de la autoestima pública (medios y redes) con voz, rostro, historia y estética propia. Dicho vulgarmente: así como el sistema de salud pública aumenta la felicidad colectiva y la autoestima de una sociedad, ser reconocido y estar en las pantallas mediáticas es condición para la felicidad y la autoestima del sujeto en esta sociedad del espectáculo.(Rincón, 2015, p. 36)

El salto discursivo dado en la cita es realmente espectacular, según este texto de producción relativamente reciente: la salud pública y el aparecer en la pantalla son puestos en el mismo rango, en tanto ambos tienen que ver con "la autoestima del sujeto". Algo así como poner en el mismo nivel el sistema educativo y sus efectos sociales, con la cuestión de los gustos estéticos sobre ropas.

No sólo cabe señalar que la función del sistema de salud está bastante lejos de ser la de producir autoestima en la población: es de asumir que hablar

2 Esta cuestión está más largamente expuesta en Follari, R. (2003). 
del sistema de salud obligaría a hablar -en homólogo nivel- del sistema de producción, distribución y consumo de mensajes mediáticos, no de la cuestión de cuál es la reacción subjetiva que este pueda promover. Pero aún si se quería hablar de esto último, correspondía entonces haber hablado del tipo de efectos subjetivos que se produce en los sistemas de salud, los cuales para nada podrían reducirse a la curiosa idea de que "aumentan la autoestima de una sociedad"; sin dejar de advertir que la autoestima es una portación de los sujetos, de modo que habría que explicar cómo se alarga ese concepto a "la sociedad" como conjunto, que además aparece indiferenciado.

Este señalamiento que hacemos no pretende ser un ensañamiento, sino la mostración de un tipo de discurso que no en cualquier ciencia social se estaría dispuesto a sostener o legitimar. ¿Por qué en comunicación se traslapan los análisis de forma tan abrupta, que pasan del sistema de salud a la mirada autosatisfecha de la propia fotografía en la red social? ¿por qué pudo en algún momento aceptarse como obvio que los medios carecían de peso en la constitución de significación social, según una lectura simplificada pero dominante de la obra de Martín-Barbero? ¿por qué se pudo tomar como cuasievidente que ya no había ciudadanía, para que creyéramos en el improbable traslado de las funciones de los ciudadanos a los consumidores, hace dos décadas atrás? (García Canclini, 1995).

"Llegó el siglo XXI. Todo parece haber cambiado (ideologías, tecnologías, democracia, jóvenes). Todo muta, menos los discursos de la comunicación, que siguen hablando de audiencias, imperialismos, representaciones, poderes, buenos y malos" (Armando. \& Rincón. 2015, p. 5).

¿No tiene algo de aquella absurda clasificación zoológica de Jorge Luis Borges, la enumeración en un mismo plano de "jóvenes" con "democracia" o "ideologías"? ¿Pertenecen en algún sentido a la misma "clase” lógica? Pero sobre todo, En tanto cambiaron las tecnologías y/o los jóvenes ¿no se puede hablar más de imperialismo?

Evidentemente, poner en el mismo nivel los cambios culturales de los jóvenes con la subsistencia o no del imperialismo implica notoria ligereza a la vez que pérdida de escala en el análisis, las que se advierten a la vez en el plano epistémico y en el ideológico-político. Pero cabe reflexionar que esta pintoresca mezcla de niveles de referencia, así como también de objetos del discurso, se hace posible a partir de la indefinición de objeto de la teoría de la comunicación, así como de la posibilidad de hablar allí desde disciplinas diversas, lo cual promueve un amplio espacio de indeterminación a partir del cual la estipulación de los límites del "discurso legítimo en el campo", tal cual la planteara Bourdieu, se hace prácticamente inviable.

Es también desde allí que se pudo establecer durante más de dos décadas a pleno la hegemonía - aún no desaparecida del todo- de los "estudios culturales" (EC) en la Comunicología latinoamericana, a nivel tanto de figuras consagradas, como de establecimiento de planes de estudio, o de bibliografía 
considerada relevante. En tanto la cultura tiene que ver con la comunicación se pudo superponer por completo sus respectivos campos de referencia, como si ambas cuestiones fueran co-extensivas conceptualmente (Follari, 2002, p. 83) ${ }^{3}$. También la economía, la sociología y la Teoría política (y no sólo ellas) tienen que ver con la comunicación, y sin embargo nadie pretendería que los análisis que se hacen para la Ciencia Económica, son válidos "sin más" como análisis en Teoría de la Comunicación; y menos aún, que además de ser válidos, fueran "suficientes"; es decir, que cubrieran "por sí" el objeto de análisis de lo comunicacional.

Hemos hecho en otra parte la crítica de los EC, que también han ensayado otros autores (Follari. \& Reynoso., 2000). Lo cierto es que la confusión sobre la identidad disciplinar, proveniente de los múltiples lenguajes que surgen de las disciplinas que proveen a Comunicación, llevó a "encontrar una identidad" de la comunidad comunicológica latinoamericana, en el plano de lo cultural. Ese predominio es el que advertimos aún, cuando puede interpretarse que los cambios en los estilos de vida juveniles, impiden que pueda hablarse de imperialismo. Obviamente que puede discutirse si la categoría de imperialismo es aún vigente para entender el presente socioeconómico planetario; pero sin dudas que esa discusión nada tiene que ver con la problemática de las culturas juveniles, y mucho con la teoría económica y política, singularmente en sus alcances en cuanto a relaciones internacionales.

\section{De qué hablar ahora}

A nivel de objeto de análisis, de vuelta estamos de la época en que se abdicó de estudiar a los medios. La insistencia en las mediaciones -cualquiera sea el significado que se otorgue a esa categoría- ${ }^{4}$, no puede ya disimular el peso de los medios en la constitución de significados dentro de la sociedad contemporánea, su aporte a la configuración de nuevas subjetividades, más su importancia para los conflictos políticos que se viven en el mundo, y singularmente en la región.

Ciertamente que los medios se han ido fusionando y complejizando, y la convergencia de los mismos es hoy una realidad, pudiendo una sola pantalla incluir Internet, televisión y mensajes telefónicos, lo cual se verifica -ademásen el uso cotidiano de los celulares de múltiples funciones por gran parte de la población. Esta realidad deja atrás cualquier melancólica actitud que deprecie los medios, en virtud de la existencia de las llamadas "comunidades de interpretación". Estas no han desaparecido, ni han perdido vigencia; pero está claro que el mundo de la palabra compartida cara a cara es hoy tributario

3 Desarrollado en Follari, R. (2002), p.83 y ss.

4 Katrine Boaventura, por entonces tesista en la Univ. de Brasilia bajo dirección de Luiz Martino, mostró claramente algunas inconsistencias en la obra de Martín-Barbero, que incluían el difuso uso de la categoría de mediaciones. Tal tesis se denominó "Recepcao e estudos culturais: uma relacao pouco discutida", Facultade de Comunicacao, Brasilia, 2009 
de lo que sucede en las redes, y estas -a su vez- guardan una relación de ida y vuelta con los medios, que las proveen a la vez que reciben material de las mismas.

A su vez, los medios han operado como la principal oposición a los gobiernos progresistas (técnicamente, varios de ellos se pueden caracterizar como "populistas") que la región ha podido sostener en las últimas dos décadas. El déficit de la comunicología para hacerse cargo de la importancia de la cuestión es llamativo. No ha habido suficiente descripción de lo que sucede, y menos aún articulación con la teoría política para proponer opciones democráticas que salvaguarden la voluntad ciudadana del bombardeo de mensajes unilaterales que unos pocos propietarios pueden fácilmente hacer sobre el conjunto de la ciudadanía. Hay mucho que pensar al respecto, mucho que proponer; pero muy poco pensado y dicho. La apelación interminable de los grandes propietarios a la libertad de prensa como derecho absoluto y que les pertenecería sin responsabilidades asociadas, no ha encontrado clara respuesta en la defensa del mayoritario derecho ciudadano a una información plural y veraz, así como a la discusión de cómo establecer una intervención estatal que lo garantice y se enfrente a los monopolios y oligopolios de las grandes cadenas mediáticas (no una intervención gubernamental sino propiamente "estatal", que supere los períodos de vigencia de un determinado gobierno).

Y por cierto la urgencia de asumir lo político en teoría de la comunicación deviene de necesidades ya no sólo surgidas desde el análisis del objeto, sino también de los sujetos epistémicos, como ciudadanos e intelectuales comprometidos, que tienen la obligación moral de ser leales a los intereses colectivos mayoritarios y a la emancipación social como horizonte histórico. En ese marco, los EC se vieron por bastante tiempo enfrentados con la economía política de la comunicación: y es esta una línea sumamente enriquecedora del análisis de lo comunicacional en el subcontinente, habiendo permitido hacer un mapeo de la propiedad y llegada de los medios que resulta muy útil para poner en evidencia las estrategias de sus propietarios para promover hegemonismo informacional, semiótico y discursivo; es decir, estrategias de hegemonismo político-ideológico.

Pero el análisis más propiamente ligado a la teoría política se ha hecho poco presente en la discusión comunicológica. Lo aportado por Laclau, y la producción de muchos politólogos que remiten a su obra, han tenido escaso peso en la "textualidad" y los debates en teoría de la comunicación. La centralidad otorgada a lo cultural ha colaborado en ese sentido, al margen de que sea una dimensión que tiene que estar presente, pero debiera hacerlo sin opacar a otras.

Advertir, entonces, que lo mediático es un aspecto central del objeto de nuestros estudios e investigaciones, nos lleva a recuperar el sitial adonde se da hoy buena parte de la lucha ideológico-política en que están embarcados nuestras ciudadanías y nuestros pueblos. Es una decisión epistemológica de anchas consecuencias hacia el plano de lo político, si bien los medios guardan 
también dimensiones de otro orden: culturales, estéticas, económicas, que también merecen ser consideradas.

Pero sólo estaremos a la altura del tiempo histórico si comenzamos a dar respuesta a las necesidades del debate político en que está imbuida la región. Por una parte, porque ese debate se da principalmente en lo mediático, y allí los sectores populares (y los gobiernos que responden a intereses populares) están en franca debilidad y situación defensiva. Y también por una razón de orden teórico-conceptual: como entreviera Lukács, lo político puede entenderse no como "una instancia más" de las varias que en la sociedad existen -según la versión que en algún momento asumiera Althusser-, sino la relación de fuerzas que ordena a todas las instancias a la vez, a su orden y hegemonía mutuas, e incluso -en una cierta parte- a las hegemonías internas que se dan dentro de cada una de tales instancias.

Esa reflexión sobre "lo político" requiere, además, no pocas modulaciones cuando la ponemos en curso en relación a las modalidades de ejercicio concreto que encontramos en la Latinoamérica contemporánea, así como a los principales discursos que alcanzan circulación dentro del ámbito de "los intelectuales", en el sentido ampliado de esta categoría.

Es Laclau quien insistió con claridad en que la política -entendida en un significado más restringido pero también fecundo- es aquel campo de comportamientos, agrupaciones y acciones que operan en relación con el manejo del poder del Estado;es decir, de la lucha por la imposición de un particular como universal, tal como había teorizado Gramsci (Laclau, 2008). Tal postura no goza de suficiente aceptación dentro de las academias de ciencias sociales, si se la compara con aquellas que apelan a la idea de autonomía de los movimientos sociales, las que proponen alguna forma de "politicidad" pensada a partir de la organización de los mismos.

Los movimientos sociales gozan de un charm difícil de combatir por aquellos que reivindican la especificidad de lo político en cuanto a la búsqueda de llegar al control del Estado. Es que sin dudas la "estatalidad" se hace altamente criticable desde el discurso mismo de Marx, en tanto aparece como una forma de alienación de la voluntad colectiva en un espacio institucional que se objetiva "por fuera y encima" de aquellos que quedan concernidos por su poder (Marx, 1967). Antes del capitalismo no se había dado la diferenciación de la sociedad en instancias separadas entre sí que guardan distintas modalidades de legitimación: lo religioso, lo jurídico, lo político, lo ético, lo estético, lo científico. Al constituirse lo político en un espacio especializado, quienes ocupan este lugar pasan a decidir sobre el conjunto social y en su nombre, pero ciertamente están "representando" a esa sociedad. Y con todas las críticas que el post-estructuralismo bien pudo hacer a finales del siglo XX a la idea de "representación", es evidente que ésta en el mejor de los casos no puede darse sin resto y por ello es siempre un tanto fallida, además de que a menudo dista de ejercerse siquiera con tal voluntad de ser "correa de transmisión" efectiva de 
las demandas sociales de aquellos a los que se supone estar representando. $\mathrm{Si}$ a ello se agrega la" crisis de lo político" que se dio sobre todo en Europa a fines del siglo XX, donde a partir de la globalización capitalista todos los programas de diferentes agrupaciones y partidos se hicieron casi equivalentes entre sí (y por ello la política apareció como un lujo caro e innecesario), podemos advertir que la desconfianza respecto del" sistema político" reconoce larga data y múltiples razones para sostenerse.

Advertidos de esta condición de fondo, sin embargo afirmaremos que es un camino sin salida el de pretender "obviar lo político" por su reducción a lo social. Porque en esta segunda acepción de lo político (diferente de la que señalamos basándonos en Lukacs, referida a aquello que ordena al conjunto de las prácticas sociales), esto no se identifica con la sociedad en su conjunto, sino con una especial actividad dentro de ella, que compromete determinados y específicos sujetos, instituciones, mediaciones y actividades. Y es un espacio donde fácilmente se puede asumir que quienes lo ocupan son "sucios, feos y malos", dado que nunca logran conformar la (imposible) plena realización de los deseos e intereses de sus demandantes, y a la vez se hacen sospechosos de detentar privilegios inmerecidos y obtenidos por vías no siempre transparentes. No suele suceder lo mismo con los empresarios, cuyo poder está más escondido y es más de largo plazo: estos no están sujetos a elecciones periódicas ni a evaluaciones externas, de modo que suelen mantenerse por tiempos mucho más prolongados. Pero no tienen la antipática tarea de decidir en nombre de terceros, sobre todo cuando tales decisiones pueden -aún en el mejor de los gobiernos- perjudicar a determinados sectores sociales (no hay modo de beneficiar a algunos de estos, sin que se implique problemas o perjuicios para otros). De tal manera, el espacio de la política está condenado a la antipatía o -en los pocos casos en que políticas de corte popular hacen mayoritaria la simpatía- a tener que renovar permanentemente los lazos de aceptación por parte de la sociedad.

Además, el neoliberalismo ha hecho de la política un mal: no es el Estado, sería el mercado el regulador "natural" de las relaciones sociales. La aparición del Estado sería la de una especie de aparato parásito que impide el libre equilibrio de los factores sociales y los sujetos en el mercado, para "distorsionarlo" en favor de algunos de manera arbitraria. Los mejores ciudadanos serían aquellos que en el mercado son capaces de lograr éxito: producir intervención estatal para que exista redistribución de recursos sería perjudicar a los mejores, condenarlos a sufrir ellos las consecuencias de la práctica de los fracasados y los incapaces, quienes se aprovecharían del acceso a beneficios que no están en consonancia con su inhabilidad intrínseca para obtenerlos por sí mismos de manera autónoma en el espacio laboral.

Como se ve, no pocas razones hay para que suela rechazarse lo estatal, y se lo haga desde posiciones ideológicas muy diversas. Pero lo cierto es que sólo desde allí, desde la disputa por el Estado, puede producirse" hegemonía”. Es a partir de la llegada al Estado que un bloque social diferente al dominante puede 
llevar adelante una tarea sistemática contra la explotación y la dominación. La apelación a los movimientos sociales -que ha tenido y tiene un enorme éxito en la Latinoamérica actual- pretende obviar este paso decisivo, y supone que desde lo social mismo puede "saltarse" a lo político, sin distinguir la singularidad del espacio que esto último tiene.

Es que en toda la trama de la sociedad hay manifestaciones de poder, y en su puesta al análisis consistió el decisivo aporte de Foucault. Sin dudas que se ejerce modos de dominación y supremacía -siempre en dinámica agonística, nunca unidireccionales- en el mundo de la sexualidad, de la escuela, de la familia, de la vida de barrio, y así sucesivamente. No cabe dudas que el poder está presente de una manera permanente y acuciosa en todos los intersticios de lo social, y que por tanto, la referencia estructural a los poderes" macro" que remiten exclusivamente a la economía y al Estado, es insuficiente para captar esa cantidad de dispositivos, discursos y prácticas diversificadas.

Pero a contrario sensu, nadie podría creer seriamente que la constitución de una alternativa a lo dominante en el campo de lo político, pueda ser una especie de suma o combinación de lo que surja de la lucha contra esos poderes "micro". No es con muchas minucias como lo estructural se entiende y modifica: no hay reducción de, lo micro a lo macro-social, pero tampoco la contraria. De tal manera, cabe enfrentar la "moda ideológica" muy presente en Latinoamérica hoy, según la cual lo propiamente político puede estar cubierto por los llamados "movimientos sociales" (ecologistas, feministas, defensores de derechos humanos, agrupaciones de defensa étnica, etc.), suponiendo que estos bastan por sí mismos para enfrentar las políticas del capitalismo contemporáneo.

Esta posición se afinca en lo que algunos han llamado pachamamismo (propio de grupos ecologistas que suponen un retorno al idealizado modo de una sociedad arcaica de agricultores y recolectores) y también del emparentado imaginario de los abyayalistas que creen que debemos retornar al mundo del Tahauntinsuyo incaico o a modalidades de la civilización maya, para así asumir la supuesta" pureza" de sociedades que no habrían estado contaminadas por la sociedad occidental y que -por ello-estarían alejadas de los males impuestos por el capitalismo.

Muy bien ha desarrollado Castro-Gómez (2019) una reciente crítica de esta posición, con la cual previamente estuvo emparentado (aunque sin haber nunca asumido ese tipo de radicales y problemáticas consecuencias). La posición por él desarrollada es muy precisa, en cuanto a mostrar que no hay origen alguno a la cual retornar, y que la modernidad no es monocorde, de modo que no debiera ser expulsada por completo, a partir de su parentesco con el capitalismo. Es la modernidad misma la que ha provisto las condiciones para la emancipación social. Y la insistencia en el universalismo que esa modernidad ha sostenido, puede ser separada de la "falsa universalización" europeizante, para ser pensada en relación con la "constitución de hegemonía" en nuestras sociedades. Es decir, en la constitución de un bloque de sectores de clase, etnias, géneros, etc., que 
dispute la tenencia del aparato del Estado a las elites dominantes, y a partir de allí construya una condición general que enfrente a la dominación, diferente del imaginario de una lucha parcial que daría cada uno de los movimientos sociales a partir de sus específicas reivindicaciones, o de la idea de una combinación de estos movimientos entre sí, que supuestamente podrían potenciarse o crear sinergia mutuamente.

Esta última idea la hemos criticado en otro espacio, en relación con la obra de Boaventura de Sousa Santos y su noción de "ecología de saberes" (Follari, 2019). La creencia en la posibilidad de que diferentes grupos culturales, etnias o colectivos reivindicatorios se liguen entre sí por vía de una traducción mutua nos resulta no sólo empíricamente inviable, sino pensada en términos que no responden a la problemática propiamente política. La relación de diversos sectores sociales con la hegemonía no se da a través de "entender al otro" horizontalmente, sino de poner reivindicaciones propias en una cadena "equivalencial" (Laclau, 2008) que atiende a llenar un significante vacío, que propone lo universal por encima de cada singularidad. De tal modo, no se trata de mirar al otro, sino de mirar con el otro hacia más allá de ambos. Eso permite superar la particularidad de cada uno, la cual no se salva con la traducción y el entendimiento, sino sólo con la constitución de un "interés común" que esté por encima de los intereses particulares de cada sector interviniente.

De tal manera, el "olvido de lo político" que encontramos en Comunicación, no es cualquier olvido; quizá se pueda hacer una analogía con el heideggeriano "olvido del ser", frente al cual el brillo de los entes operaba sólo como una tapadera respecto de aquello que efectivamente vale la pena pensar. Lo político establece la forma del ordenamiento social, que implica incluso jerarquizar y subordinar -si bien de manera sólo parcial, acorde a lo dicho- muchos de los poderes microsociales que en una sociedad se producen. Si seguimos renunciando a ponernos en la centralidad del debate que nos convoca en tanto especialistas que queremos ser de la cuestión comunicación, que incluye prioritariamente a lo mediático, y con ello al lugar de los medios como poder político que nadie elige pero se ejerce con suma vigencia, estaremos dejando de ofrecer alguna específica contribución al presente momento de la historia latinoamericana, que no es cualquiera-dado los enfrentamientos ideológicos que pueblan a sus concretos actores políticos-. Y en tal caso, estaremos condenados a la inanidad histórica, así como a un lugar secundario en la consideración dentro del conjunto de las ciencias sociales en la región.

Este articulo es una versión modificada y aumentada de la Conferencia presentada al Primer Seminario internacional de la Red Latina de Teoría Crítica en Comunicación y Cultura, "Crisis, capitalismo y transformación: los desafíos de las teorías críticas”, CIESPAL, Quito, julio de 2017 


\section{Referencias bibliográficas}

Amado, A. \& Rincón, O. (2015). Introducción. En: Rincón, O. \& Amado, A. La comunicación en mutación, Friedrich Ebert Stiftung, Bogotá. (versión en Internet)

Bourdieu. P. et al. (1975). El oficio de sociólogo. Siglo XXI, Buenos Aires.

Castro-Gómez, S. (2019). El tonto y los canallas (notas para un republicanismo transmoderno). Univerisdad Javeriana, Bogotá.

Kuhn, T. (1980). La estructura de las revoluciones científicas. F.C.E., México. . (1989). Conmensurabilidad, comparabilidad y comunicabilidad. En: Kuhn, T. (1989). ¿Qué son las revoluciones científicas? y otros ensayos. Paidós/I.C.E., Barcelona.

Follari, R. (2000). Sobre la inexistencia de paradigmas en las ciencias sociales. En Follari, R. (2000). Epistemología y sociedad, Homo Sapiens, Rosario.

. (2002), Teorías débiles: para una crítica de la deconstrucción y de los estudios culturales. Homo Sapiens, Rosario.

. (2003), La moldura en espejo: encrucijadas epistemológicas de las Ciencias de la Comunicación, Tram(p)as de la Comunicación. Núm.16. Universidad Nacional de La Plata . (2019). Boaventura de Sousa Santos: de la dispersión al orden en la ecología de saberes. En: Utopía y praxis latinoamericana. Núm.86. Universidad de Zulia, Maracaibo, jul.-set.

García Canclini, N. (1995). Consumidores y ciudadanos (conflictos multiculturales de la globalización). Grijalbo, México.

Laclau. E. (2008). La razón populista. Fondo de Cultura Económica, Buenos Aires.

Martino, L. (2007). Teorias da comunicacao: ¿̨muitas o poucas? Atelié ed., Cotia (SP).

Marx, C. (1967). Crítica de la filosofía del derecho de Hegel. Ed. Grijalbo, México.

Reynoso, C. (2000). Apogeo y decadencia de los estudios culturales: una visión antropológica. Gedisa, Barcelona.

Rincón, O. (2015). Lo popular en la comunicación: culturas bastardas + ciudadanías celebrities. En: Rincón, O. \& Amado, A. La comunicación en mutación. Friedrich Ebert Stiftung, Bogotá. (versión en Internet) 
